\title{
CONDICIONES MÍNIMAS Y ACREDITACIÓN DE ALTA CALIDAD, UNA VISIÓN NACIONAL E INSTITUCIONAL
}

Juanita Benavides Delgado ${ }^{1}$

Como medida para asegurar la calidad de la Educación Superior y sus implicaciones en la gestión académica en la Universidad de Ciencias Aplicadas y Ambientales U.D.C.A, se presenta una revisión cronológica de la legislación pertinente expedida en Colombia, a raíz de la Constitución Política, de 1991, en lo relativo a la función de inspección y de vigilancia, a cargo del gobierno nacional, para estas instituciones.

\section{MARCO LEGAL}

La citada Constitución establece en el Artículo 67 que "la Educación es un derecho de la persona y un servicio público que tiene una función social garantizando, además, la autonomía universitaria, las libertades de enseñanza, aprendizaje, investigación y cátedra y asignándole al Estado la facultad de regular y ejercer la suprema inspección y vigilancia de la educación, con el fin de velar por su calidad". Posterior a la reforma de 1991, se expide la Ley 30 de 1992, conocida como la ley de Educación Superior que señala el ejercicio de la inspección y vigilancia por parte del Estado (Capítulo VII, Artículo 31,32 y 33).

El gobierno decide reglamentar lo relativo al aseguramiento de alta calidad de los programas de pregrado a través de la Ley 30, que creó el Sistema Nacional de Acreditación para garantizar a la sociedad que los programas académicos y las instituciones de Educación Superior que se someten voluntariamente a este sistema y sean acreditados, llenen los más altos requisitos de calidad y cumplan sus propósitos y sus objetivos.

La Reglamentación sobre la Acreditación Voluntaria de Alta Calidad para los programas académicos de pregrado, se consolida en distintas normas así:

1 Directora Departamento de Planeación. Universidad de Ciencias Aplicadas y Ambientales U.D.C.A. Economista Especialista en Estadística. jbenavid@udca.edu.co
- Decreto 2904 de 1994, define el proceso, que se inicia con la auto evaluación, continua con la evaluación efectuada por Pares Académicos y culmina con el acto de acreditación proferido por el Ministerio de Educación Nacional, previo concepto del Consejo Nacional de Acreditación CNA.

- Acuerdo No. 4 del 28 de marzo de 1995 el Consejo Superior de la Educación Superior CESU, creado mediante Artículo 34 de la Ley 30 de 1992, que pone en funcionamiento el Sistema Nacional de Acreditación, reglamentando las funciones e integración del CNA.

- Acuerdo No.6 del 14 de diciembre de 1995 que establece las políticas generales que rigen la acreditación en Colombia, inscritas en el contexto del fomento de la calidad.

Con la expedición de la Ley 30 , se produjo a nivel nacional una proliferación de programas, de tal forma que entre 1990 y 1996 su número aumentó en un 60\% y el número de instituciones, en un $11 \%$ (Cifuentes $\mathcal{E}$ Pérez, 1999), lo cual llevó a la comunidad académica a cuestionar la calidad de los programas que se ofrecían en Colombia.

Las primeras acciones para garantizar las mínimas condiciones de calidad de los programas académicos aparecen en función de los programas de postgrado. Es así, como el decreto 2791 del 22 de diciembre de 1994, define las condiciones mínimas para la aprobación de un programa de doctorado.

Posterior a la reglamentación relacionada con la acreditación voluntaria de alta calidad de los programas de pregrado, aparece la necesidad de las Asociaciones de Profesionales, para establecer las condiciones que garantizaran una mínima calidad de los mismos apoyando, de esta forma, las funciones de inspección y de vigilancia ejercida por el gobierno nacional. Este 
proceso, se inicia con los programas de pregrado y especialización en Educación, basado en la Ley 115, Ley General de la Educación, donde se ratifica el deber del Estado por atender permanentemente los factores que favorecen la calidad y el mejoramiento de la educación, la calificación y formación de educadores de la más alta calidad científica y técnica.

En este sentido, en 1998 mediante el Decreto 272, se establece la necesidad de mantener un mejoramiento continuo de la calidad de los docentes a través de la Acreditación Previa, como mecanismo para que todos los programas de pregrado y de especialización en Educación cumplieran, de manera obligatoria, con los requisitos de calidad señalados por el Consejo Nacional de Acreditación. Para el caso de programas de Maestría y Doctorado, la certificación de la Acreditación Previa sería expedida por el Ministerio de Educación Nacional previo concepto de la entonces Comisión Nacional de Maestrías y Doctorados, con base en los lineamientos trazado por el CESU.

Al esfuerzo por garantizar la calidad mínima de los programas en Educación, se une el de otras asociaciones de profesionales que conduce a la expedición, por parte del Ministerio de Educación Nacional, de los Decretos 792 y 917 del 22 de mayo de 2001, los cuales establecen el estándar de calidad para los programas de Pregrado en Ingeniería y Ciencias de la Salud. El Registro Calificado al que hace referencia estos decretos y que obtienen los programas que cumplen dichos estándares, era otorgado por el Ministerio de Educación Nacional previo concepto del CNA. Dicho Registro Calificado es válido por siete años y su actualización se debería tramitar ante el Instituto Colombiano de Fomento de la Educación Superior ICFES.

Para ese entonces, año 2001, el proceso de acreditación voluntaria de alta calidad continuaba en el país para los programas académicos de pregrado, en todos sus niveles, consolidándose así una cultura de la evaluación de la calidad de la Educación Superior. En junio de este año, el CNA expide los lineamientos para la Acreditación Institucional, en un momento en que la acreditación alcanzaba reconocimiento, en el ámbito nacional, mostrando el compromiso de las instituciones frente al servicio público. Este tipo de acreditación busca ser garante de la calidad de las instituciones que la alcancen y corre, paralelamente, a la de programas.
Como en los programas de Educación, Ciencias de la Salud e Ingeniería, las Asociaciones de Profesionales establecieron con el Ministerio de Educación Nacional, la necesidad de cumplir con estándares de calidad para la creación y el funcionamiento de todos los programas a nivel de pregrado.

Para el año 2002, existían los procesos de Acreditación Voluntaria de programas de pregrado y el cumplimiento de las condiciones mínimas de algunos programas, cuya finalidad, según el ICFES, era intrínseca al mejoramiento de la calidad de la Educación Superior y se hizo necesario señalar que los programas de pregrado, acreditados voluntariamente, no tendrían que adelantar el proceso de verificación de cumplimiento de estándares de calidad para obtener el Registro Calificado.

Ante esta situación, donde cada asociación de profesionales presionaba por su propia reglamentación, el gobierno nacional expide el Decreto 2566 de octubre 9 de 2003, en el cual se establecieron la condiciones de calidad para el ofrecimiento de programas académicos en general, independiente del nivel de formación de cada uno de ellos, por lo tanto, se debían cumplir para programas a nivel de técnico profesional, tecnológico universitario, especialización, maestría y doctorado.

Cronológicamente, se observa que el principal énfasis del Gobierno, se centró en los procesos de acreditación de alta calidad a principios de la década de los 90's. Este proceso, se caracteriza por ser eminentemente voluntario, sin efectos punitivos, mientras que el registro calificado, que se obtiene con el cumplimiento de los estándares de calidad, de carácter obligatorio, implica que la institución no puede ofrecer los programas que carecen del mismo, llevando incluso al cierre de aquellos que se presenten sin dicho requisito.

La acreditación solo se puede adelantar cuando un programa académico esté en funcionamiento y tenga varias promociones de egresados, mientras que el Registro Calificado, se debe obtener como condición para la apertura de un nuevo programa.

Ambos procesos son de carácter temporal. La Acreditación tanto de programas académicos como institucional oscila entre tres y diez años; el Registro Calificado, para programas de Pregrado en todos sus niveles, es por siete años y para los programas de Postgrado, es por cinco años. 


\section{LA U.D.C.A FRENTE A LA ACREDITACIÓN Y EL REGISTRO CALIFICADO DE PROGRAMAS}

Una vez creado el sistema de acreditación en Colombia, la U.D.C.A presentó, en el año 1997, tres programas al proceso de acreditación voluntaria: Medicina Veterinaria, Zootecnia e Ingeniería Agronómica.

Los doctores José Revelo Revelo (q.e.p.d), Carlos Augusto Hernández R. y como coordinador, Ramsés Hakim Murad, integraron la comisión que visitó a la U.D.C.A, para la verificación de condiciones institucionales.

Para este primer ejercicio, con miras a la acreditación voluntaria de programas, la U.D.C.A estableció la cultura de la autoevaluación comenzando una capacitación a todas las directivas sobre el modelo planteado por el CNA. Se dispuso de una sesión semanal del Consejo Académico dedicado a la lectura y al análisis de cada una de las características e indicadores que conformaban los sietes factores iniciales. Posteriormente, se establecieron dos franjas institucionales enfocadas a la autoevaluación en los tres programas, garantizando la participación de todos los miembros de la comunidad universitaria.

Luego de remitidos los respectivos documentos, donde se consignaron los ejercicios participativos de autoevaluación, el CNA seleccionoó los pares académicos, quienes luego de las visitas correspondientes, emitieron un informe que fue analizado por el CNA, en el marco de la evaluación integral, para finalmente recomendar al Ministerio la acreditación voluntaria del programa de Medicina Veterinaria, la cual se consolidó con la expedición de la Resolución No.1284, del 8 de junio de 1999 del Ministerio de Educación Nacional. A la fecha, la situación de la U.D.C.A frente a este proceso se puede observar en la tabla 1.

Para el año 2001 y como consecuencia de la expedición de la reglamentación sobre condiciones mínimas de calidad expedida por el Ministerio de Educación Nacional, se inició el trabajo para la obtención del registro calificado de cuatro programas: Ingeniería Geográfica e Ingeniería Comercial y Medicina y Enfermería, respectivamente.

Tabla 1. Acreditación Voluntaria de Alta Calidad. Programas acreditados U.D.C.A.

\begin{tabular}{|c|c|c|c|c|c|c|}
\hline PROGRAMA & RESOLUCIÓN & FECHA & VIGENCIA & RESOLUCIÓN & FECHA & VIGENCIA \\
\hline $\begin{array}{c}\text { Medicina } \\
\text { Veterinaria }\end{array}$ & 1284 & $\begin{array}{c}\text { Junio } 8 \text { de } \\
1999\end{array}$ & 3 años & 1565 & $\begin{array}{c}\text { Julio } 01 \text { de } \\
2003\end{array}$ & 6 años \\
\hline Zootecnia & 481 & $\begin{array}{c}\text { Marzo } 7 \text { de } \\
2003\end{array}$ & 5 años & $\begin{array}{c}\text { En proceso de } \\
\text { reacreditación }\end{array}$ & & \\
\hline $\begin{array}{c}\text { Ingeniería } \\
\text { Agronómica }\end{array}$ & 1630 & $\begin{array}{c}\text { Julio } 17 \text { de } \\
2002\end{array}$ & 3 años & 1226 & $\begin{array}{c}\text { Mayo } 16 \text { de } \\
2007\end{array}$ & 4 años \\
\hline M.V.Z. & 2364 & $\begin{array}{c}\text { Junio } 17 \text { de } \\
2005\end{array}$ & 3 años & $\begin{array}{c}\text { En proceso de } \\
\text { reacreditación }\end{array}$ & & \\
\hline $\begin{array}{c}\text { Ciencias del } \\
\text { Deporte }\end{array}$ & 3423 & $\begin{array}{c}\text { Agosto } 18 \text { de } \\
2005\end{array}$ & 7 años & & & \\
\hline
\end{tabular}

Para el año 2008, los programas de pre y postgrado, ofrecidos por la U.D.C.A, que cuentan con Registro Calificado, se relacionan en la tabla 2 . En proceso de obtención del Registro Calificado se encuentran los programas de especialización: Mejoramiento Animal, Gestión Social y Ambiental y Manejo Sostenible del Sistema Suelo, agua y planta en el trópico.
La U.D.C.A actualmente cuenta con el $15 \%$ de sus programas acreditados, el $76 \%$ con registro calificado y el $9 \%$ en proceso para obtención de este último.

Para la U.D.C.A es clara la búsqueda permanente de la excelencia académica, la cual está explícita en su MISIÓN Institucional y está íntimamente ligada al proceso de 
Tabla. 2. Relación de Programas ofrecidos por la U.D.C.A con Registro Calificado.

\begin{tabular}{|c|c|c|c|}
\hline PROGRAMA & RESOLUCIÓN & FECHA & VIGENCIA \\
\hline Ing. Comercial & 5350 & Mayo 26 de 2006 & 7 años \\
\hline Química Farmacéutica & 2425 & Mayo 26 de 2006 & 7 años \\
\hline Química & 2530 & Mayo 30 de 2006 & 7 años \\
\hline Ciencias Ambientales & 7483 & Nov.24 de 2006 & 7 años \\
\hline Medicina & 5912 & 05 de octubre de 2007 & 7 años \\
\hline Enfermería & 5911 & 05 de octubre de 2007 & 7 años \\
\hline Esp. Laboratorio Clínico $-\mathrm{P}^{*}$ & 3826 & Julio 14 de 2006 & 7 años \\
\hline Esp. Laboratorio Clínico. - - ${ }^{* *}$ & 3827 & Julio 14 de 2006 & 7 años \\
\hline Esp. Sanidad Animal -P & 3825 & Julio 14 de 2006 & 7 años \\
\hline Esp. Sanidad Animal -D & 621 & Feb. 13 de 2007 & 7 años \\
\hline Nutrición Animal -P & 4326 & Agosto 2 de 2006 & 7 años \\
\hline Nutrición Animal -D & 4324 & Agosto 2 de 2006 & 7 años \\
\hline Producción Animal -P & 4527 & Agosto 10 de 2006 & 7 años \\
\hline Producción Animal -D & 4323 & Agosto 2 de 2006 & 7 años \\
\hline Reproducción Bovina Tropical -P & 4327 & Agosto 2 de 2006 & 7 años \\
\hline Epidemiologia Vet. P & 4325 & Agosto 2 de 2006 & 7 años \\
\hline Entrenamiento Deportivo & 3889 & Julio 10 de 2007 & 7 años \\
\hline Economía & 3416 & Junio 21 de 2007 & 7 años \\
\hline Contaduría Pública & 3415 & Junio 21 de 2007 & 7 años \\
\hline Administración de Empresas & 3368 & Junio 20 de 2007 & 7 años \\
\hline Negocios Internacionales & 2069 & Mayo 2 de 2007 & 7 años \\
\hline Mercadeo & 3369 & Junio 20 de 2007 & 7 años \\
\hline Finanzas & 6926 & Noviembre 132007 & 7 años \\
\hline $\begin{array}{l}\text { Esp. de enfermería en Atención } \\
\text { Domiciliaria }\end{array}$ & 6828 & Noviembre 9 de 2007 & 5 años \\
\hline MVZ -CARTAGENA & 1500 & 26 de Marzo de 2008 & 7 años \\
\hline
\end{tabular}

\section{* P: Modalidad Presencial.}

** D: Modalidad a Distancia

autoevaluación y, por ende, al de autoregulación. La reglamentación que en esta materia ha expedido el gobierno nacional, desde el año 1991, ha permitido fortalecer estos procesos en la universidad $y$, ha demostrado a la sociedad en general, la calidad de sus programas y el compromiso institucional frente a la función social. En síntesis, se puede asegurar que los resultados derivados de los programas de aseguramiento de la calidad, representados en la Acreditación Voluntaria y el Registro Calificado de programas, se evidencian en la institución.
Tratar de hacer un balance sobre los resultados de estos procesos, no es fácil, pero se podrá sintetizar en los siguientes diez aspectos, todos ellos de especial importancia:

Un primer logro está en el Reconocimiento Institucional como Universidad, alcanzado después de diez años de iniciado el proceso, con la expedición de la Resolución 49765 del 29 de diciembre de 2004, que llevó a la adopción de nuevos estatutos, con la Resolución No.802 del 11 de marzo de 2005, del Ministerio de Educación Nacional. 
En segundo lugar, la consolidación de las funciones sustantivas en la Institución. En investigación, con líneas, con grupos clasificados por COLCIENCIAS y con proyectos de investigación co-financiados por entidades del orden nacional e internacional. En Extensión, con el trabajo con comunidades y entidades del sector público y privado, a nivel nacional e internacional. En Docencia, con la consolidación de una planta docente que garantice una formación de calidad y la adopción de un estatuto profesoral que, además de señalar derechos y deberes, establece un escalafón que incentiva a los profesores a cualificarse con formación a nivel de postgrado aumentando, de esta forma, la producción académica.

El papel protagónico de la U.D.C.A, en el ámbito internacional, se puede considerar como un tercer logro y se evidencia a través de los programas de internacionalización que incluye la participación en las redes de Educación Superior, a nivel Iberoamericano.

El cuarto éxito alcanzado, se evidencia en la consolidación y en el crecimiento de la infraestructura física de la institución contando, actualmente, con un campus de once hectáreas y dos predios adicionales en el barrio parcelación El Jardín (sede El Remanso y Sede Oriental), lo que constituye una extensión significativa en el patrimonio de la Universidad. La nueva planta física ofrece espacios dedicados al Bienestar Social Universitario, que garantizan mejores condiciones a todos los miembros de la comunidad universitaria. El quinto logro, se evidencia en la cantidad y la calidad de los recursos que apoyan las funciones sustantivas en la Universidad; se incluyen recursos bibliográficos, de laboratorio y de informática, entre otros.

La organización administrativa en la institución, se evidencia como un sexto avance, al registrar, permanentemente, las acciones de los cuerpos colegiados, mediante actas periódicas, con la participación de los estamentos de la institución y la revisión continua de la estructura orgánica que favorezca el trabajo en equipo, haciéndola cada vez menos jerárquica y más horizontal.

El aumento de la cobertura de los programas constituye el séptimo logro de la U.D.C.A, al pasar de dos programas académicos de pregrado, en el año 1983 a 18 programas de pregrado y 14 especializaciones en el año 2008 y de 110 estudiantes, en 1983, a 3100, en el presente año.
La madurez académica alcanzada por la U.D.C.A, en sus 25 años de existencia, se evidencia en los procesos de autorregulación, que parten de la autoevaluación integral y ética adelantada, a nivel institucional y, en particular, en cada dependencia, lo que constituye el octavo logro.

El impacto de los egresados de la U.D.C.A, quienes han logrado dejar en alto el nombre de su Alma Mater, con su desempeño profesional, tanto nacional como internacionalmente, constituyen un noveno éxito a resaltar.

Como décimo logro, se identifica uno de tipo misional relacionado con las acciones adelantadas por la universidad en favor del desarrollo sostenible y el compromiso frente al ambiente, desde una perspectiva compleja.

Solamente un estricto, continuo y permanente proceso de autoevaluación permitiría el crecimiento sostenible de la Universidad y el reconocimiento de la calidad de la misma por organismos nacionales e internacionales y por la sociedad civil, en función de los cuales se ofrece el servicio público cultural en nuestro país.

Mas allá del cumplimiento de la normativa expedida por el gobierno nacional en función del aseguramiento de la calidad de la educación superior, la excelencia académica en la U.D.C.A, seguirá guiando la gestión institucional basado en un sólido, permanente y continuo proceso de autoevaluación y el consecuente proceso de autoregulación.

\section{BIBLIOGRAFÍA}

DEPARTAMENTO NACIONAL DE PLANEACIÓN. Conpes de la Educación Superior. Mayo 1995

CONSEJO SUPERIOR DE LA EDUCACIÓN SUPERIOR CESU. Acuerdo 4. Marzo 1995

CONSEJO SUPERIOR DE LA EDUCACIÓN SUPERIOR CESU. Acuerdo 6. Diciembre 1995

CIFUENTES, J.; PÉREZ, M.D. 1999. Sistema Colombiano de Acreditación: la búsqueda de un mejor modo de hacer Universidad. Cuadernos Ascun, Santa Fe de Bogotá, No.7, p.29-51. 
CONSEJO NACIONAL DE ACREDITACION. 2001. Lineamientos para la Acreditación Institucional. Serie Documento CNA No.2 Bogotá.

CONSTITUCIÓN POLÍTICA DE COLOMBIA. 2007.

MINISTERIO DE EDUCACIÓN NACIONAL. Ley 30 de 1992.

MINISTERIO DE EDUCACIÓN NACIONAL. Ley 115 de 1994.

MINISTERIO DE EDUCACIÓN NACIONAL. 1994. Decreto 2904.

MINISTERIO DE EDUCACIÓN NACIONAL. 22 de Diciembre de 1994. Decreto 2791.
MINISTERIO DE EDUCACIÓN NACIONAL 20 de Agosto de 1996. Decreto 1475.

MINISTERIO DE EDUCACIÓN NACIONAL. 11 de Febrero de 1998. Decreto 272.

MINISTERIO DE EDUCACIÓN NACIONAL. 22 de Mayo de 2001. Decretos 916 y 917.

MINISTERIO DE EDUCACION NACIONAL. 20 de Diciembre de 2001. Decreto 2802.

MINISTERIO DE EDUCACIÓN NACIONAL. 10 de Mayo de 2002, Decretos 936,937, 938, 939 y 940.

Recibido: Abril 15 de 2008

Aceptado: Mayo 12 de 2008 